\title{
Effect of Temperature and Band Nonparabolicity on Density of States of Two Dimensional Electron Gas
}

\author{
G. Gulyamov', P. J. Baymatov ${ }^{2}$, B. T. Abdulazizov ${ }^{2}$ \\ ${ }^{1}$ Namangan Engineering-Pedagogical Institute, Namangan, Uzbekistan \\ ${ }^{2}$ Namangan State University, Namangan, Uzbekistan \\ Email: bt_abdulazizov@mail.ru
}

Received 25 November 2015; accepted 22 February 2016; published 25 February 2016

Copyright (C) 2016 by authors and Scientific Research Publishing Inc.

This work is licensed under the Creative Commons Attribution International License (CC BY). http://creativecommons.org/licenses/by/4.0/

(c) (7) Open Access

\begin{abstract}
The analysis of the density of states for electrons in single quantum well, the conduction band nonparabolicity take is account. It is shown that the degree of conduction band nonparabolicity pronounces depending on the energy density of states. With increasing temperature, a step change in the density of states smoothes and at high temperatures is completely blurred. Nonparabolicity dispersion law manifests itself in a wide range of temperatures. Calculations are carried out for the example of the quantum wells in InAs and InSb.
\end{abstract}

\section{Keywords}

Quantum Well, Density of States, Band Nonparabolicity, Thermal Broadening

\section{Introduction}

Recently, investigations of narrow band gap semiconductors InAs, InSb [1]-[3] based heterostructures have been paid much attention because they are essential to developing semiconductor devices. In these structures, the conduction band is strongly nonparabolicity. Properties of subband nonparabolicity of a two dimensional electron gas (2DEG) can be studied by the cyclotron resonance experiments. In heterostructures InAs/AlSb, a quantum well (QW) depth for electrons reaches up to $\sim 1.3 \mathrm{eV}$. Modern technology makes it possible to obtain a structure with a 2DEG with a concentration of up to $n_{s} \approx 10^{13} \mathrm{~cm}^{-2}$ [4]. Depending on the width of the QW, one or more subbands may be formed in the system.

It is known that with an increase electron concentration $n_{\mathrm{s}}$ when the Fermi level crosses the bottom of the next series subbands, the density of state (DOS) of 2DEG drastically changes. In case of parabolic band energy 
dependence of DOS is abruptly changing rectangular steps [5]. Nonparabolicity can affect the structure of the resulting subbands in the QW, and can shift the bottom of the subbands [6]. Therefore, it should be manifested in the energy dependence of the DOS of 2DEG. With increasing temperature, due to thermal broadening of the levels, as well as the temperature dependence of the band gap of $E_{g}$ (the degree of nonparabolicity directly depend on $E_{g}$ ), the energy dependence of the DOS of 2DEG in general is complex.

The purpose of this work is to obtain expressions for the DOS of 2DEG with allowance conduction band nonparabolicity and graphical analysis of the expressions. To study the effect of temperature on the DOS, the calculations will be carried out numerically for heterostructures of InAs and InSb based QW.

\section{Electron Energy Spectrum in QW}

In the simplest approximation, the nonparabolic energy spectrum of the 2DEG can be represented as

$$
\chi(E)=E_{\|}+E_{n}
$$

Here, $E_{\|}=\hbar^{2} k_{\|}^{2} / 2 m(0), \quad k_{\|}^{2}=k_{x}^{2}+k_{y}^{2}$, and $m(0)=m_{n}$ the effective mass at the bottom of the conduction band. In approaching an infinitely high barrier

$$
E_{n}=\frac{\hbar^{2} k_{n}^{2}}{2 m(0)}=\frac{\hbar^{2} \pi^{2} n^{2}}{2 m(0) L^{2}}=E_{1} n^{2}, k_{n}=k_{z}=\frac{\pi n}{L}
$$

In real situations, the energy levels $E_{n}$ and form of the function $\chi(E)$ can determine the 8-zone $\mathbf{k} \cdot \mathbf{p}$ hamiltonian [7] [8] considering the effects of the finite height of the barrier, the deformation caused by the mismatch of the lattice constant, etc.

The dependence of $\chi(E)$ is a complex in the Kane model [9]. However, the majority of cases it is possible to apply various approximations. For simplicity, below we use the following approximation [10]

$$
\chi(E)=E\left(1+\alpha E+\beta E^{2}\right), \alpha=\frac{1}{\varepsilon_{g}}\left(1-\frac{m_{n}}{m_{0}}\right)^{2}, \beta=-\frac{2}{\varepsilon_{g}^{2}} \frac{m_{n}}{m_{0}}\left(1-\frac{m_{n}}{m_{0}}\right)
$$

where, $m_{0}$-free electron mass, $\varepsilon_{g}$-band gap of the bulk semiconductor. The energy is measured from the bottom of the conduction band of the bulk semiconductor. The parameters used in the calculations for InAs, InSb are shown in Table 1.

According to (1-3), bottom $n$-th subband $k_{\|}=0$ is determined from the equations

$$
\chi(E)=E\left(1+\alpha E+\beta E^{2}\right)=E_{n}
$$

Solving Equation (4), it is possible to determine of interband transition energy, for example $E_{12}=E_{2}-E_{1}$. Figure 1 shows the results of calculations $L$ dependence of $E_{12}$ for single InAs QW. The same figure also shows the results of experimental studies on the energy of intersubband transitions $E_{12}$ [11]-[13].

The figure shows that a simple model (4) gives qualitatively correct results only for wide quantum wells. For example, in QW width $L=15 \mathrm{~nm}$ value obtained from (4) to the bottom of the first and second subband respectively equal to 0.0636 and $0.2 \mathrm{eV}$. Hence, estimated intersubband transition energy is $E_{12}=0.1364 \mathrm{eV}$, an

\begin{tabular}{|c|c|c|}
\hline \multicolumn{3}{|c|}{$\mathrm{T}=4.2 \mathrm{~K}$} \\
\hline Compounds & $\mathrm{InSb}$ & InAs \\
\hline$\varepsilon_{g}, \mathrm{eV}$ & 0.237 & 0.42 \\
\hline$m_{n} / m_{0}$ & 0.014 & 0.023 \\
\hline$\alpha, \mathrm{eV}^{-1}$ & 4.1 & 2.27 \\
\hline$\beta, \mathrm{eV}^{-2}$ & -0.5 & -0.26 \\
\hline
\end{tabular}
experimental measured value is $0.134 \mathrm{eV}$ [13]. With a decrease in $L$ results obtained by the model (4) strongly deviates from the experiment. This deviation may be due to the finiteness of the barrier height $(\sim 1.36 \mathrm{eV})$, the

Table 1. The parameters used in the calculations for InAs, InSb. 


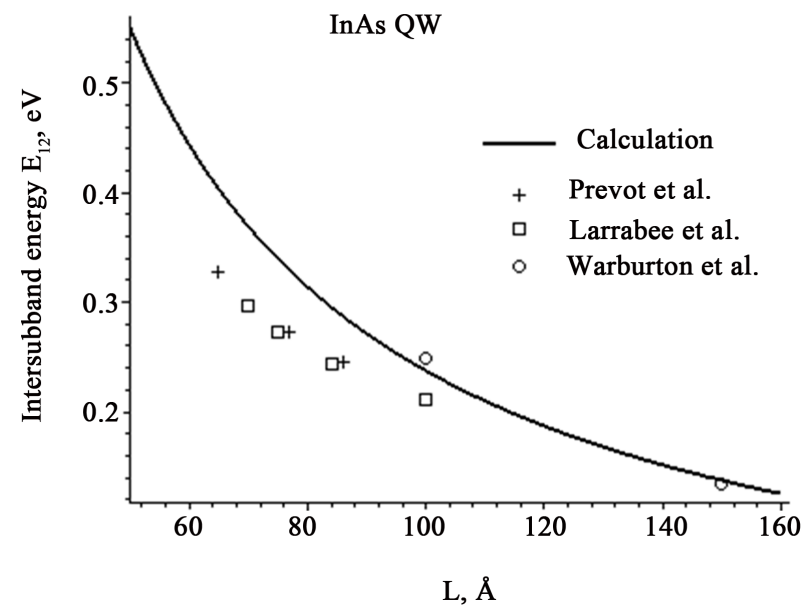

Figure 1. Intersubband energy $\mathrm{E}_{12}$ for the InAs single QW. The crosses represent the experimental data [11], squares [12], circles [13] and the solid line-Equation (4).

deformation of the structure due to mismatch of the lattice constant, and the effect of depolarizing shift unaccounted for in the model (4). Therefore, following a study of DOS carried out for the QW width $L=15 \mathrm{~nm}$ on the example of InAs and InSb.

\section{DOS at Zero Temperatures}

Knowing the energy spectrum of the electron gas can determine its DOS. To determine the DOS of 2DEG, we use the equation of the total number of particles. After summation over the spin degree it has the form

$$
N=2 \frac{L_{x} L_{y}}{(2 \pi)^{2}} \sum_{n=1}^{\infty} \int \mathrm{d} k_{x} \mathrm{~d} k_{y} f(E)
$$

where $f(E)$-Fermi-Dirac distribution function.

Writing $\mathrm{d} k_{x} \mathrm{~d} k_{y}$ to polar coordinates, expressing $k_{\|}^{2}$ according to (1) through a total energy $E$ and taking into account, that $\chi(E)-E$ is positive, we have

$$
N=\frac{L_{x} L_{y}}{\pi} \frac{m(0)}{\hbar^{2}} \sum_{n=1}^{\infty} \int_{0}^{\infty} \frac{\partial \chi(E)}{\partial E} f(E) \Theta\left[\chi(E)-E_{n}\right] \mathrm{d} E
$$

where, $\Theta(x)$-Heaviside function. From (6) we obtain the equation for the two dimensional concentration

$$
n_{2 D}=\frac{N}{L_{x} L_{y}}=\frac{m(0)}{\pi \hbar^{2}} \sum_{n=1}^{\infty} \int_{0}^{\infty} \frac{\partial \chi(E)}{\partial E} f(E) \Theta\left[\chi(E)-E_{n}\right] \mathrm{d} E=\int_{0}^{\infty} N(E) f(E) \mathrm{d} E
$$

Hence, we obtain the formula for DOS of 2DEG, where band nonparabolicity (3) is taking into account

$$
N(E)=N_{0} \sum_{n=1}^{\infty} \frac{\partial \chi(E)}{\partial E} \Theta\left[\chi(E)-E_{n}\right]
$$

or explicitly

$$
N(E)=N_{0} \sum_{n=1}^{\infty}\left(1+2 \alpha E+3 \beta E^{2}\right) \Theta\left[E\left(1+\alpha E+\beta E^{2}\right)-E_{n}\right]
$$

Here

$$
N_{0}=\frac{m_{n}}{\pi \hbar^{2}}=\frac{m_{n}}{m_{0}} \frac{0.413 \times 10^{15}}{\mathrm{eV} \cdot \mathrm{cm}^{2}}, E_{1}=\frac{\hbar^{2} \pi^{2}}{2 m_{n} L^{2}}=\frac{\pi^{2} 3.81 \mathrm{eV}}{\left(m_{n} / m_{0}\right)(L / \AA)^{2}}=\frac{37.6 \mathrm{eV}}{\left(m_{n} / m_{0}\right)(L / \AA)^{2}}
$$


In the particular case when $\alpha=\beta=0$, from (9) follows known formula for the DOS corresponding parabolic dispersion [5]

$$
N(E)=N_{0} \sum_{n=1}^{\infty} \Theta\left(E-E_{n}\right)
$$

Formula (9) can be rewritten as

$$
N(E)=N_{0} \sum_{n=1}^{\infty} \frac{m(E)}{m_{n}} \Theta\left[E\left(1+\alpha E+\beta E^{2}\right)-E_{n}\right]
$$

where

$$
m(E)=m_{n}\left(1+2 \alpha E+3 \beta E^{2}\right)
$$

is energy-dependent electron effective mass. It is also obtained from the definitions of the transport mass

$$
\frac{1}{m_{\|}(E)}=\frac{1}{\hbar^{2}} \frac{1}{k_{\|}} \frac{\partial E}{\partial k_{\|}}
$$

taking into account the spectrum (1) and (3).

Figure 2 and Figure 3 shows the energy dependence of the DOS for the QW width $L=15 \mathrm{~nm}$ appropriately InAs, InSb, and calculated by the Formula (9).

From these graphs follows that the band nonparabolicity can be lead to the following results:

-The bottom of each subbands moves down as compared to the parabolic case.

-This is clear from the fact that the bottom of the subbands is inversely proportional to the effective mass of $m(E)$.

-The height of the jumps increases with energy, since it is proportional to $m(E)$.

-Within each subbands the DOS increases linearly.

According to (9), the change of the DOS within each subbands must be square.

However, this amendment to the plot is weak, as for the structures of InAs, InSb the approximation $\beta=0$ is also satisfactory.

\section{DOS at Finite Temperatures}

In letter [14] it is shown that the temperature dependence of the DOS can be described decomposition $N(E, T)$ to the number of GN-function (defined as energy derived probability of filling the energy levels). In [15], study using the DOS expansion in GN-functions able to explain the temperature dependence of the DOS in quantizing magnetic fields. This expansion can be written as

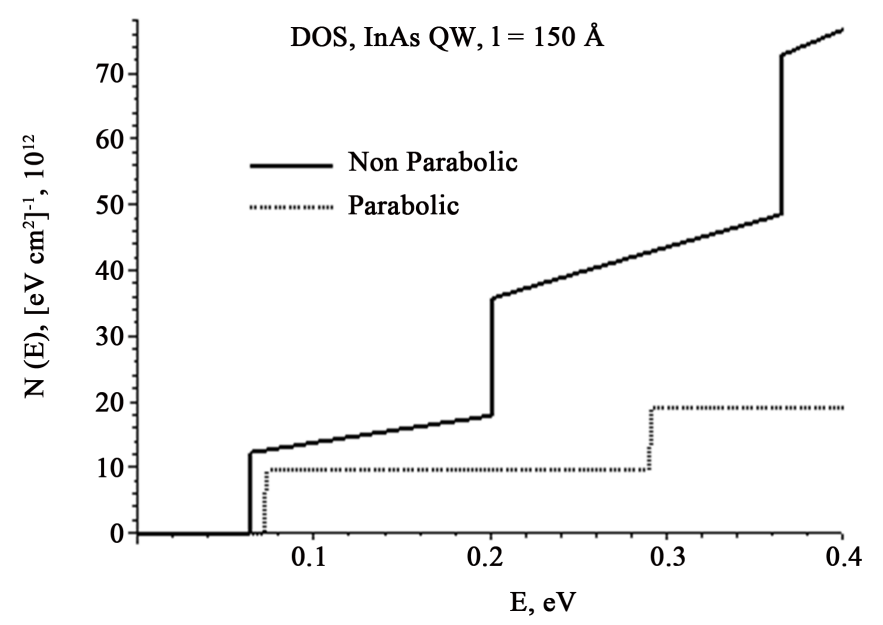

Figure 2. DOS for the InAs single QW, $L=150 \AA, T=0 \mathrm{~K}$. The solid line represents the nonparabolic and dotted line-the parabolic approximation. 


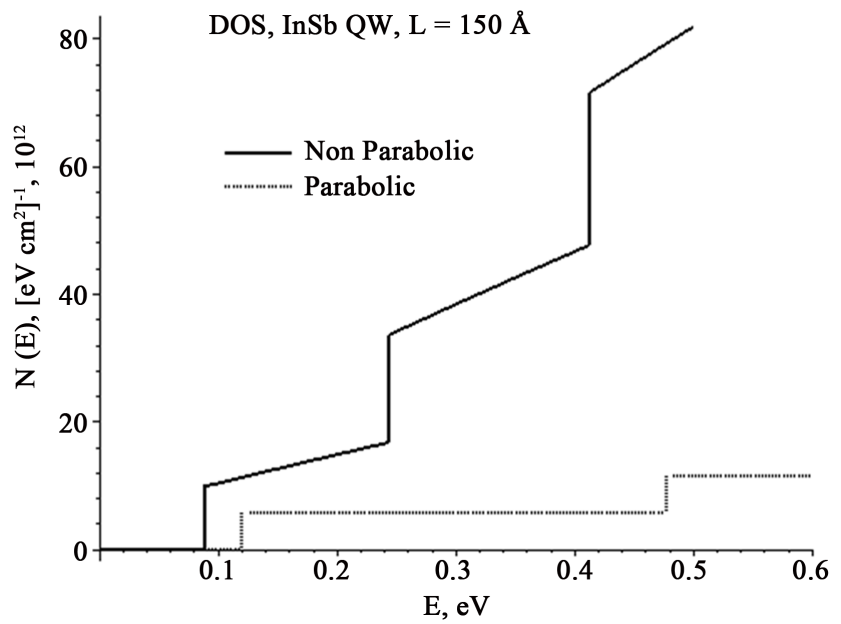

Figure 3. DOS for the InSb single QW, $L=150 \AA, T=0 \mathrm{~K}$. The solid line represents the nonparabolic and dotted line- - the parabolic approximation.

$$
N(E, T)=\int_{0}^{\infty} N\left(E^{\prime}, 0\right) G N\left(E^{\prime}, E, T\right) \mathrm{d} E^{\prime}
$$

where

$$
G N\left(E^{\prime}, E, T\right)=\frac{1}{T} \exp \left[\frac{E^{\prime}-E}{T}-\exp \left(\frac{E^{\prime}-E}{T}\right)\right]
$$

Using (9) (14) and (15) $N(E, T)$ can be plotted for specific QW. Figure 4 and Figure 5 shows the energy dependence of $N(E, T)$ must for the QW width $L=15 \mathrm{~nm}$ appropriately InAs, InSb, and calculated according to the Formula (14) for any temperatures.

As seen from Figure 4 and Figure 5 the temperature greatly affects the shape depending $N(E, T)$. When low temperature the thermal generated electrons from the energy states is weak and it does not lead to a strong broadening of the energy levels and the DOS has sharply stepped shape. At the same angles of steps slightly smoothed. There dependency nonparabolicity dispersion law remains the same as Figure 2 and Figure 3. The low temperature has small effect on the energy form of DOS. With further increase of temperature the thermal generated electrons from the energy levels increased. This leads to a strong broadening thermodynamic DOS. It significantly changes its shape. In this case, the thermal broadening almost washed away a step change in the DOS and rectangular steps turned into a smooth blend smooth curve.

At low energies steps completely disappear. With increasing energy, appear slight deviations to the provisions of the former steps of the respective discrete levels. With further increase of the energy-the thermodynamic DOS become a smooth curve, but nonparabolicity dispersion law manifests itself in a wide range of temperatures.

\section{Conclusions}

In this paper, the analysis of the density of states of the 2DEG in a single quantum is well. The calculations are performed for narrow-gap semiconductors InAs and InSb.

The nonparabolicity of conduction band leads to the following features of DOS:

-The bottom of each subband shifted downwards compared with parabolic case (Figure 2 and Figure 3);

-The height of the jumps increases with energy, since it is proportional to $m(E)$;

-Within each subband DOS increases linearly;

-With increasing temperature step change DOS smoothed and high temperatures completely blurred (Figure 4 and Figure 5). 


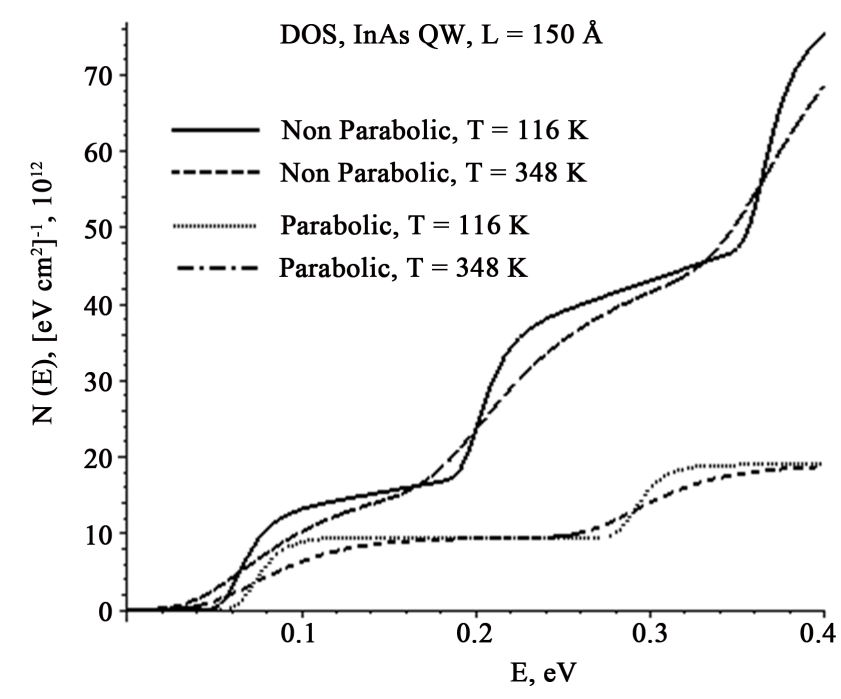

Figure 4. DOS for InAs single QW, $L=150 \AA$ at nonzero temperatures.

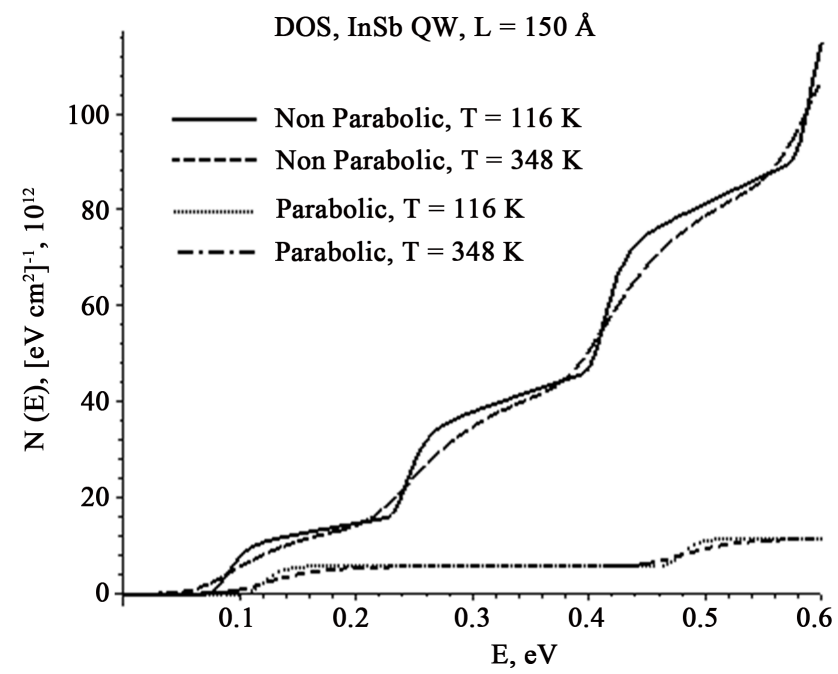

Figure 5. DOS for InSb single QW, $L=150 \AA$ at nonzero temperatures.

\section{Acknowledgements}

This work was supported by the Scientific and Technical program Republic of Uzbekistan (Grant F2-OTO-15494).

\section{References}

[1] Vasilyev, Y.B., Gouider, F., Nachtwei, G. and Buckle, P.D. (2010) The Cyclotron Resonance in Heterostructures with the InSb/AlInSb Quantum Wells. Semiconductors, 44, 1511. http://dx.doi.org/10.1134/S1063782610110266

[2] Spirin, K.E., Kalinin, K.P., Krishtopenko, S.S., Maremyanin, K.V., Gavrilenko, V.I. and Sadofyev, Y.G. (2012) Features of the Persistent Photoconductivity in InAs/AlSb Heterostructures with Double Quantum Wells and a Tunneling-Transparent Barrier. Semiconductors, 46, 1396. http://dx.doi.org/10.1134/S1063782612110206

[3] Barate, D., Teissier, R., Wang, Y. and Baranov, A.N. (2005) Short Wavelength Intersubband Emission from Quantum Cascade Structures. Applied Physics Letters, 87, Article ID: 051103. http://dx.doi.org/10.1063/1.2007854

[4] Aleshkin, V.Y., Gavrilenko, V.I., Ikonnikov, A.V., Sadofyev, Y.G., Bird, J.P., Jonhson, S.R. and Zhang, Y.-H. (2005) Cyclotron Resonance in Doped and Undoped InAs/AlSb Heterostructures with Quantum Wells. Semiconductors, 39, 62-66. http://dx.doi.org/10.1134/1.1852647 
[5] Shik, A.Y., Bakueva, L.G., Musikhin, S.F. and Rykov, S.A. (2001) Fizika nizkorazmernih system. SPb, Nauka, 160 p. (In Russian)

[6] Ekenberg, U. (1989) Nonparabolicity Effects in a Quantum Well: Sublevel Shift, Parallel Mass, and Landau Levels. Physical Review B, 40, 7714-7726. http://dx.doi.org/10.1103/PhysRevB.40.7714

[7] Winkler, R. (1996) Cyclotron Resonance and Subband-Landau Level Coupling in 2D Electron and Hole Gases. Surface Science, 361-362, 411-414. http://dx.doi.org/10.1016/0039-6028(95)00433-5

[8] Foreman, B.A. (1997) Elimination of Spurious Solutions from Eight-Band $k \cdot p$ Theory. Physical Review B, 56, 642648.

[9] Kane, E.O. (1957) Band Structure of Indium Antimonide. Journal of Physics and Chemistry of Solids, 1, 249-261. http://dx.doi.org/10.1016/0022-3697(57)90013-6

[10] Ridley, B.K. (1999) Quantum Processes in Semiconductors. Clarendon Press, Oxford.

[11] Prevot, I., Vinter, B., Julien, F.H., Fossard, F. and Marcadet, X. (2001) Experimental and Theoretical Investigation of Interband and Intersubband Transitions in Type-II InAs/AlSb Superlattices. Physical Review B, 64, Article ID: 195318. http://dx.doi.org/10.1103/PhysRevB.64.195318

[12] Larrabee, D.C., Khodaparast, G.A., Kono, J., Ueda, K., Nakajima, Y., Nakai, M., Sasa, S., Inoue, M., Kolokolov, K.I., Li, J. and Ning, C.Z. (2003) Temperature Dependence of Intersubband Transitions in InAs/AlSb Quantum Wells. Applied Physics Letters, 83, 3936. http://dx.doi.org/10.1063/1.1626264

[13] Warburton, R.J., Gauer, C., Wixforth, A., Kotthaus, J.P., Brar, B. and Kroemer, H. (1996) Intersubband Resonances in InAs/AlSb Quantum Wells: Selection Rules, Matrix Elements, and the Depolarization Field. Physical Review B, 53, 7903. http://dx.doi.org/10.1103/PhysRevB.53.7903

[14] Gulyamov, G. and Sharibaev, N.Y. (2011) Determination of the Density of Surface States of the Boundary Semiconductor-Insulator Interface in a MIS Structure. Fizika and Technika Poluprovodnikov, 45, 178. (In Russian)

[15] Gulyamov, G., Erkabaev, U.I. and Sharibaev, N.Y. (2014) Vliyanie Temperaturi na termodinamicheskuyu plotnost sostoyaniy v kvantuyushem magnitnom pole. Fizika and Technika Poluprovodnikov, 48, 1323. (in Russian) 